\title{
Procedural Anxiety Subscale
}

National Cancer Institute

\section{Source}

National Cancer Institute. Procedural Anxiety Subscale. NCI Thesaurus. Code 157702.

A subscale of the Pediatric Quality of Life Brain Tumor Module designed to assess issues associated with procedural anxiety. 